\title{
Six-Month Pulmonary Impairment after Severe COVID-19: A Prospective, Multicentre Follow-Up Study
}

\author{
Paola Faverio $^{a}$ Fabrizio Luppi $^{\mathrm{a}}$ Paola Rebora $^{\mathrm{b}}$ Sara Busnelli ${ }^{\mathrm{a}}$ Anna Stainer $^{\mathrm{a}}$ \\ Martina Catalano $^{a} \quad$ Luca Parachini $^{a} \quad$ Anna Monzani $^{a}$ Stefania Galimberti ${ }^{b}$ Francesco Bini $^{c}$ \\ Bruno Dino Bodinic Monia Betti ${ }^{\mathrm{d}}$ Federica De Giacomi ${ }^{\mathrm{d}}$ Paolo Scarpazza elisa Oggionni $^{\mathrm{e}}$ \\ Alessandro Scartabellati $^{f}$ Luca Bilucaglia ${ }^{f}$ Paolo Cerutig Denise Modina ${ }^{g}$ Sergio Harari ${ }^{\text {h }}$ \\ Antonella Caminati ${ }^{i} \quad$ Maria Grazia Valsecchib ${ }^{b} \quad$ Giacomo Bellani $^{j} \quad$ Giuseppe Foti $^{j}$ \\ Alberto Pesci ${ }^{a}$ \\ aschool of Medicine and Surgery, University of Milano Bicocca, Respiratory Unit, San Gerardo Hospital, ASST

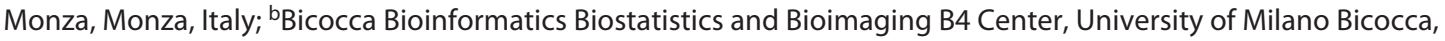 \\ Monza, Italy; 'Department of Internal Medicine, UOC Pulmonology, Ospedale G. Salvini, ASST-Rhodense, Milan,

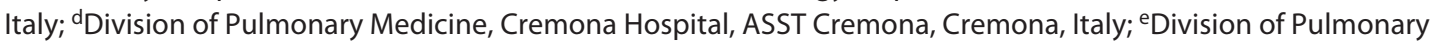

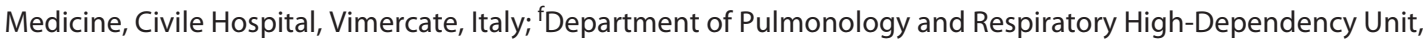 \\ Ospedale Maggiore, Crema, Italy; 9U.O. Pneumologia e Fisiopatologia Respiratoria, ASST Spedali Civili di Brescia, \\ Brescia, Italy; hepartment of Medical Sciences, Department of Clinical Sciences and Community Health, San \\ Giuseppe Hospital, MultiMedica IRCCS and Università degli Studi di Milano, Milan, Italy; 'U.O. di Pneumologia e \\ Terapia Semi-Intensiva Respiratoria, Servizio di Fisiopatologia Respiratoria ed Emodinamica Polmonare, Ospedale \\ San Giuseppe, MultiMedica IRCCS, Milan, Italy; 'Department of Anesthesia and Intensive Care Medicine, School of \\ Medicine and Surgery, University of Milano Bicocca, Monza, Italy
}

\section{Keywords}

COVID-19 • Pneumonia - Respiratory function tests ·

Pulmonary fibrosis · Radiology and other imaging ·

Follow-up

\begin{abstract}
Background: Long-term pulmonary sequelae following severe acute respiratory syndrome coronavirus 2 (SARS-CoV-2) pneumonia are not yet confirmed; however, preliminary observations suggest a possible relevant clinical, functional, and radiological impairment. Objectives: The aim of this study was to identify and characterize pulmonary sequelae caused by SARS-CoV-2 pneumonia at 6-month follow-up.
\end{abstract}

Methods: In this multicentre, prospective, observational cohort study, patients hospitalized for SARS-CoV-2 pneumonia and without prior diagnosis of structural lung diseases were stratified by maximum ventilatory support ("oxygen only," "continuous positive airway pressure," and "invasive mechanical ventilation") and followed up at 6 months from discharge. Pulmonary function tests and diffusion capacity for carbon monoxide (DLCO), 6-min walking test, chest X-ray, physical examination, and modified Medical Research Council (mMRC) dyspnoea score were collected. Results: Between March and June 2020, 312 patients were enrolled (83, 27\%

Paola Faverio and Fabrizio Luppi are joint first authors. ClinicalTrials.gov Identifier: NCT04435327.
Correspondence to:

Paola Faverio, paola.faverio@ unimib.it 
women; median interquartile range age $61.1[53.4,69.3]$ years). The parameters that showed the highest rate of impairment were DLCO and chest X-ray, in $46 \%$ and $25 \%$ of patients, respectively. However, only a minority of patients reported dyspnoea (31\%), defined as $m M R C \geq 1$, or showed restrictive ventilatory defects (9\%). In the logistic regression model, having asthma as a comorbidity was associated with DLCO impairment at follow-up, while prophylactic heparin administration during hospitalization appeared as a protective factor. The need for invasive ventilatory support during hospitalization was associated with chest imaging abnormalities. Conclusions: DLCO and radiological assessment appear to be the most sensitive tools to monitor patients with the coronavirus disease 2019 (COVID-19) during followup. Future studies with longer follow-up are warranted to better understand pulmonary sequelae.

(c) 2021 S. Karger AG, Basel

\section{Background}

The coronavirus disease 2019 (COVID-19) pandemic, caused by the severe acute respiratory syndrome (SARS) coronavirus 2 (SARS-CoV-2) and initiated in Wuhan (China) in December 2019, has expanded dramatically throughout the world during the last year [1]. Pneumonia and acute respiratory distress syndrome (ARDS) are frequent manifestations of COVID-19; its pathogenic mechanisms are not entirely known and patients develop various degrees of respiratory failure, ranging between oxygen therapy, non-invasive ventilation, and invasive mechanical ventilation (IMV). Some studies identified ARDS-like lesions, characterized by an inflammatory reaction in early phase [2], leading to fibrotic sequelae or to the development of pulmonary emphysema, evolving to lung function impairment. Prior experience from H1N1 influenza pneumonia and SARS showed that long-term pulmonary fibrosis developed in up to $10 \%$ and $4.6 \%$ of patients with ARDS-like forms, respectively $[3,4]$. Some more recent observations also highlighted the association between excessive distention of pulmonary parenchyma during IMV, leading to baro-volutrauma, and the development of post-ARDS pulmonary fibrosis $[5,6]$.

These previous observations may suggest a potentially relevant impact of pulmonary sequelae after severe $\mathrm{CO}$ VID-19; however, preliminary reports after 3 months of follow-up of these patients showed conflicting results [79], suggesting the importance to continue the follow-up of these patients. Furthermore, some of the short-term follow-up pulmonary sequelae, including ground-glass opacities and atelectasis, require longer observation to assess whether they will be irreversible and their potential impact on pulmonary function. Furthermore, results from large, multicentre prospective cohort studies with 6 and 12 months follow-up are in the majority of cases still ongoing [10]. This study aims to identify and characterize pulmonary sequelae, in patients hospitalized for SARSCoV-2 pneumonia, at 6-month follow-up after hospital discharge, and to evaluate their association with the maximum ventilatory support received during hospitalization.

\section{Materials and Methods}

\section{Study Design and Participants}

In this multicentre, prospective, observational cohort study, we enrolled consecutive patients hospitalized for laboratory-confirmed SARS-CoV-2 pneumonia between March and June 2020 in 7 hospitals in Lombardy, a region of Northern Italy populated by about 10 million people: San Gerardo Hospital, Monza; G. Salvini Hospital, Garbagnate Milanese; San Giuseppe Hospital, Milan; Spedali Civili, Brescia; Ospedale Civile, Vimercate; Ospedale Maggiore, Crema; Ospedale Maggiore, Cremona. Patients were followed up at 6 months from discharge to evaluate the presence of pulmonary sequelae with clinical evaluation, complete pulmonary function tests (PFTs), including plethysmography and diffusion capacity for carbon monoxide (DLCO) with single-breath technique, 6-min walking test (6MWT), and chest X-ray. Clinical evaluation included the collection of a dyspnoea score (Modified Medical Research Council [mMRC] scale) and lung auscultation to detect the presence of pathological lung sounds.

Inclusion and exclusion criteria are summarized in Table $1 . \mathrm{Pa}-$ tients were stratified according to the maximum oxygen/ventilatory support received during hospital stay: (1) oxygen therapy alone, (2) continuous positive airway pressure (CPAP), (3) invasive mechanical ventilation (IMV). CPAP and IMV were applied according to the position papers on the management of respiratory failure in patients with COVID-19 [11].

This study received ethics committee approval (ASST Monza, 3389, May 21st, 2020) and was registered on clinicaltrial.gov (ClinicalTrials.gov Identifier: NCT04435327). The study is composed of 2 follow-up visits at 6 months and 1 year from hospital discharge. In this manuscript, we report results from the 6-month visits. All patients provided written informed consent at the time of enrolment. The study is reported according to STROBE guidelines [12].

\section{Procedures}

PFTs and DLCO measurements were performed according to the American Thoracic Society (ATS)/European Respiratory Society (ERS) standardization using a dry spirometer [13, 14]. PFT parameters were expressed as absolute and percentage of a theoretical value calculated by Global Lung Function 2012 equations [15]. 6MWT was performed according to the guidelines recommended by the ATS [16]. The lower limits of normal for distance walked in healthy men and women were calculated according to 
Table 1. Inclusion and exclusion criteria

Inclusion criteria

Age between 18 and 80 years

Diagnosis of SARS-CoV-2 infection by positive PCR on nasal-pharyngeal swab or on bronchoalveolar lavage in case of doublenegative nasal-pharyngeal swabs performed at least $24 \mathrm{~h}$ apart (GeneXpert Cepheid; InGenius Elitech; Abbott real-time SARS-CoV-2 assay Abbott; SARS-CoV-2 plus ELITe MGB ${ }^{\circ}$ assay Elitech; Allplex ${ }^{\text {Tx }}$ SARS-CoV-2 assay arrow diagnostics)

Clinical/instrumental signs of interstitial pneumonia and acute respiratory failure $\left(\mathrm{PaO}_{2} / \mathrm{FiO}_{2}<300\right.$ in room air) on hospital admission

Written informed consent

Patients were discharged at home or in another hospital facility

Exclusion criteria

Severe renal failure, defined as a glomerular filtration rate lower than $30 \mathrm{~mL} / \mathrm{min}$, upon discharge

NYHA class IV (unable to carry on any physical activity without discomfort), upon discharge

Pregnancy or breastfeeding

Bacterial and/or fungal pulmonary superinfection during hospital stay

Prior diagnosis of chronic obstructive pulmonary disease, pulmonary emphysema, pulmonary fibrosis, or bronchiectasis

$\mathrm{PaO}_{2} / \mathrm{FiO}_{2}=$ ratio of $\mathrm{PaO}_{2}$ to $\mathrm{FiO}_{2}$. Prior diagnosis of asthma and obstructive sleep apnoea syndrome were not excluded since they do not cause permanent and irreversible radiological and/or functional impairment. PCR, polymerase chain reaction; SARS-CoV-2, severe acute respiratory syndrome coronavirus 2; $\mathrm{NYHA}$, New York Heart Association; $\mathrm{FiO}_{2}$, fractional inspired oxygen; $\mathrm{PaO}{ }_{2}$, arterial oxygen partial pressure.

the equation created by Enright and colleagues [17]. Chest X-rays were evaluated by the pulmunologist and radiologist in charge for the presence of parenchymal abnormalities (reticulations, groundglass opacities, and/or consolidation) and their extension (upper, middle, and lower region for each lung). Lung auscultation was performed by a pulmonologist, and the presence of pathological breath sounds (crackles, "velcro" crackles, wheezing, rhonchi, squawks, and rales) was reported.

\section{Outcomes}

The primary end point of the study was DLCO impairment (DLCO $\%<80 \%$ of predicted) evaluated at 6 months from hospital discharge. The secondary end points of the study were also assessed at 6 months from hospital discharge and were (1) vital capacity (VC), forced vital capacity (FVC), Tiffeneau Index (FEV1/ FVC ratio), forced expiratory volume in the 1st second (FEV1), total lung capacity (TLC), and residual volume alterations; (2) dyspnoea evaluated through mMRC scale; (3) pathological lung sounds at chest auscultation; (4) radiological alterations on chest $\mathrm{X}$-ray; and (5) variation from the expected of the normal distance walked on 6MWT.

\section{Statistical Analysis}

A sample size of 360 patients was calculated to provide $80 \%$ power to detect an increase from $2 \%$ (patients who received oxygen therapy alone) to $10 \%$ (patients who received CPAP or IMV) in the percentage of pulmonary sequelae at 1 year after hospital discharge with a logistic regression model and a statistical significance of 0.05 . Assuming a $10 \%$ rate of dropout, the sample size was increased to 400 patients, equally distributed in each of the 3 arms.

In the descriptive analysis, qualitative variables have been summarized by counts and percentages, while quantitative characteristics by quartiles. Patients characteristics of the groups identified by maximum ventilatory support received were compared by Fisher's exact test and Kruskal-Wallis rank sum test, as appropriate. In order to evaluate the association between maximum ventilatory support and pulmonary sequelae, a logistic regression model was applied adjusting for predefined variables: age, gender, body mass index, cardiovascular diseases, diabetes, asthma, and treatment during hospital stay with systemic steroids or prophylactic heparin. Interactions were investigated and included in the model if statistically significant ( $p$ value $<0.05$ ). A sensitivity analysis included also smoking as potential confounder. The model was fitted on the primary end point (DLCO impairment) and on radiological alterations. Results were reported as odds ratio (OR) with $95 \%$ confidence interval (CI). The analyses were performed in R (version 4.0.4).

\section{Results}

\section{Study Population}

In the study period, 420 consecutive hospitalized patients with SARS-CoV-2 pneumonia were screened for study participation. Out of the 420, 312 (83, 27\% women; median interquartile range [IQR] age 61.1 [53.4,69.3] years) met the inclusion and exclusion criteria, provided consent and thus were enrolled in the final cohort and were stratified as follows: 71 patients in the "oxygen alone" group, 144 patients in the "CPAP" group, and 97 patients in the "IMV" group, Figure 1. Out of the 108 excluded patients, none was dead between assessment of eligibility and study enrolment. The baseline clinical features of the study population stratified by maximum oxygen/ventilatory support are shown in Table 2 . The majority of patients were never smokers, with no differences 
Fig. 1. Study flowchart. CPAP, continuous positive airway pressure; IMV, invasive mechanical ventilation.

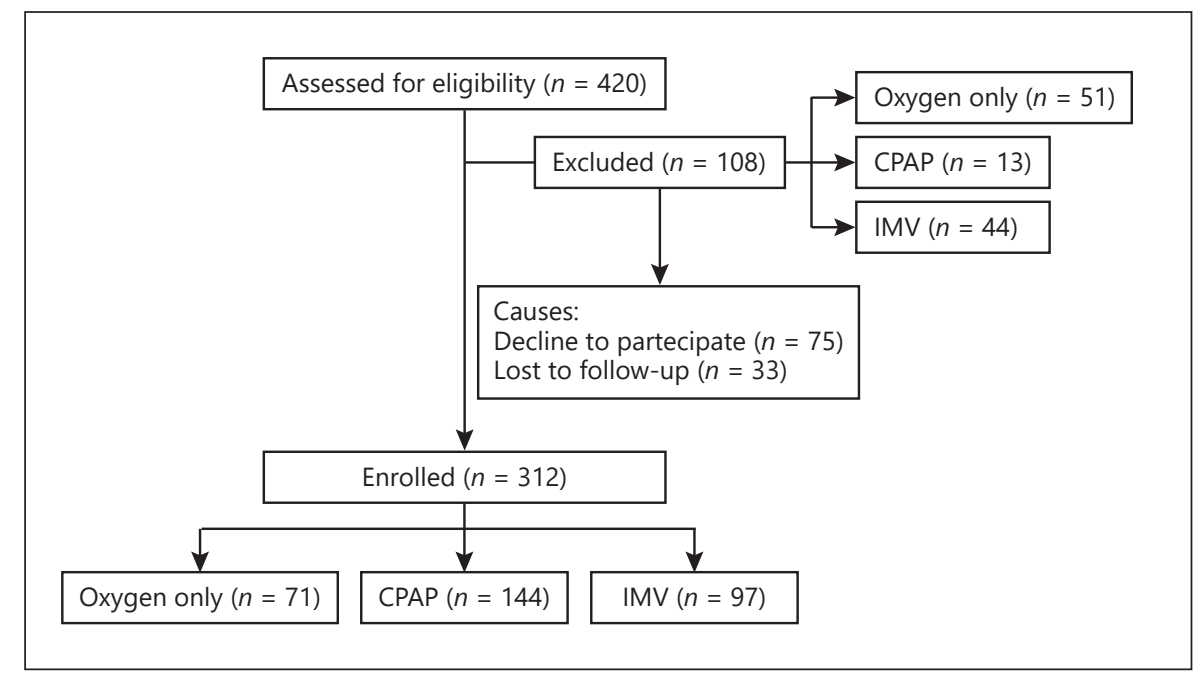

between the study groups. The most frequently encountered comorbidities were obesity (34\%), hypertension (29\%), cardiovascular diseases (22\%), and diabetes (14\%). The majority of patients showed only one or the absence of comorbidities (78\%). In regard to treatments received during hospitalization for COVID-19, patients in the "oxygen alone" group received significantly less specific treatments than the other groups. Pulmonary thromboembolism and deep vein thrombosis, 2 possible complications of COVID-19, were reported in $4.8 \%$ and $1.3 \%$ of patients, respectively, with no differences between groups.

\section{Evaluation of Pulmonary Sequelae}

In regard to the presence of DLCO impairment (primary end point), we observed a statistically significant difference between groups with the highest prevalence of DLCO alteration in the "oxygen alone" $(n=40,58 \%)$ and "IMV" group $(n=52,54 \%)$ and the lowest in the "CPAP" group $(n=50,36 \%)$, Table 3. However, patients in the "IMV" and "CPAP" group showed slightly more frequently moderate and severe DLCO impairment than the "oxygen alone" group see online suppl. Fig. 1; for all online suppl. material, see www.karger.com/doi/10.1159/000518141.

No differences between groups were observed in the other parameters of PFTs, with the exception of FVC and Tiffeneau Index, Table 3. When considering FVC as a continuous variable, patients in the "IMV" group showed lower values than "CPAP" and "oxygen only" group (median [IQR] FVC\% 102\% [91.8-112.5], 106\% [96.3-118.5], and $107 \%$ [95.2-115.8], respectively, $p$ value 0.046$)$. However, when considering pathological values of FVC\% (below $80 \%$ of predicted), only a minority of patients pre-

Six-Month Pulmonary Impairment after Severe COVID-19 sented this condition (10, 7, and $11 \%$ of cases in the "oxygen only," "CPAP," and "IMV" groups, respectively) with no differences between groups.

A minority of patients in our cohort showed a restrictive pattern using both definition based on $\operatorname{FVC}(28,9 \%)$, defined as having a normal FEV1/FVC and an FVC $<80 \%$ predicted [18], and based on TLC $(52,16.6 \%)$, defined as having a normal FEV1/VC and a reduction in TLC [19]. An obstructive pattern (defined as Tiffeneau Index $<0.7$ with a concomitant reduction of FEV1 $<80 \%$ ) was observed only in 7 (2.2\%) patients, in the majority of cases in the "oxygen only" group ( 5 cases). Among the 7 cases with obstructive pattern, 1 was active, 2 prior smokers, and 1 had asthma as comorbidity. The distribution of obstructive and restrictive ventilatory patterns in the 3 study groups is summarized in Figure 2.

Median distance walked at $6 \mathrm{MWT}$ ranged between 150 and $700 \mathrm{~m}$, with no differences between groups, Table 4 . However, up to 46 (17\%) of patients showed a distance walked lower than expected, again without differences between groups. No patients showed oxygen desaturation or required oxygen supplementation during the test.

Characterizing the degree of dyspnoea reported by patients through the mMRC scale, the majority of patients (62\%) were "not troubled by breathlessness except on strenuous exercise," with no differences between groups, Table 4 . Also at lung auscultation only a minority of patients, ranging from 4 to $12 \%$ according to study group presented pathological sounds, mostly "velcro" crackles ( 21 cases) followed by wheezing ( 5 cases). "Velcro" crackles were significantly more frequent in the "IMV" group. Among the 21 cases with "velcro" crackles at physical ex- 
Table 2. Demographics and clinical characteristics of study cohort

\begin{tabular}{|c|c|c|c|c|}
\hline Demographics & $\begin{array}{l}\text { Oxygen only } \\
(N=71)\end{array}$ & $\begin{array}{l}\text { CPAP } \\
(N=144)\end{array}$ & $\begin{array}{l}\text { IMV } \\
(N=97)\end{array}$ & $p$ value \\
\hline Age, years, median [IQR] & $61.1[53.3,71.9]$ & $61.1[53.1,67.6]$ & $60.8[55.2,68.2]$ & 0.600 \\
\hline Female gender, $N(\%)$ & $31(44)$ & $33(23)$ & $19(20)$ & 0.001 \\
\hline BMI, kg/m², median [IQR] & $27.5[24.6,31.4]$ & $28.7[26.6,31.3]$ & $28.1[25.7,31.0]$ & 0.234 \\
\hline \multicolumn{5}{|l|}{ Smoking history, $N(\%)$} \\
\hline No & $47(84)$ & $83(65)$ & $51(65)$ & \multirow{3}{*}{0.100} \\
\hline Active & $1(2)$ & $8(6)$ & $6(8)$ & \\
\hline \multirow[t]{2}{*}{ Prior } & $8(14)$ & $36(28)$ & $21(27)$ & \\
\hline & Oxygen only & CPAP & IMV & $\mathrm{p}$ value \\
\hline \multicolumn{5}{|l|}{ Comorbidities } \\
\hline Cardiovascular diseases, $N(\%)$ & $12(17)$ & $31(22)$ & $25(26)$ & 0.397 \\
\hline Hypertension, $N(\%)$ & $22(31)$ & $43(30)$ & $26(27)$ & 0.816 \\
\hline Cerebrovascular diseases, $N(\%)$ & $1(1)$ & $3(2)$ & $1(1)$ & 0.857 \\
\hline Asthma, $N(\%)$ & $9(13)$ & $4(3)$ & $4(4)$ & 0.015 \\
\hline OSAS, $N(\%)$ & $2(3)$ & $3(2)$ & $1(1)$ & 0.674 \\
\hline Chronic kidney diseases, $N(\%)$ & $4(6)$ & $2(1)$ & $3(3)$ & 0.162 \\
\hline Liver diseases, $N(\%)$ & $2(3)$ & $3(2)$ & $0(0)$ & 0.200 \\
\hline Diabetes, $N(\%)$ & $12(17)$ & $20(14)$ & $13(13)$ & 0.799 \\
\hline Autoimmune diseases, $N(\%)$ & $1(1)$ & $2(1)$ & $0(0)$ & 0.603 \\
\hline Prior cancer, $n(\%)$ & $6(8)$ & $2(1)$ & $6(6)$ & 0.026 \\
\hline \multicolumn{5}{|l|}{ No. of comorbidities, $N(\%)$} \\
\hline 0 & $24(34)$ & $68(47)$ & $39(40)$ & \multirow{4}{*}{-} \\
\hline 1 & $29(41)$ & $44(31)$ & $38(39)$ & \\
\hline 2 & $11(15)$ & $25(17)$ & $16(16)$ & \\
\hline$\geq 3$ & $7(10)$ & $7(5)$ & $4(4)$ & \\
\hline \multicolumn{5}{|c|}{ Treatments associated with COVID-19 } \\
\hline Systemic steroid, $N(\%)$ & $18(31)$ & $65(56)$ & $43(58)$ & 0.003 \\
\hline Prophylactic heparin, $N(\%)$ & $18(31)$ & $55(47)$ & $44(59)$ & 0.005 \\
\hline Tocilizumab, $N(\%)$ & $5(9)$ & $17(15)$ & $19(25)$ & 0.031 \\
\hline Remdesivir, $N(\%)$ & $1(2)$ & $2(2)$ & $12(16)$ & $<0.001$ \\
\hline Mucolytics, $N(\%)$ & $13(22)$ & $35(30)$ & $30(41)$ & 0.084 \\
\hline Hyperimmune plasma, $N(\%)$ & $0(0)$ & $1(1)$ & $1(1)$ & 1.000 \\
\hline Lopinavir/ritonavir, $N(\%)$ & $21(36)$ & $76(66)$ & $34(46)$ & $<0.001$ \\
\hline Hydroxychloroquine, $N(\%)$ & $42(72)$ & $100(88)$ & $59(80)$ & 0.042 \\
\hline
\end{tabular}

BMI, body mass index; CPAP, continuous positive airway pressure; IMV, invasive mechanical ventilation; IQR, interquartile range; OSAS, obstructive sleep apnoea syndrome; COVID-19, coronavirus disease 2019.

amination, 10 (48\%) showed abnormal chest X-ray (reticulations in 9 out of 10 cases), and 7 (33\%) had DLCO impairment, of mild entity in all patients, but none showed restrictive pattern at PFTs. Three of the 5 cases who showed wheezing also had asthma, but none of them had an obstructive ventilatory defect.

Chest X-ray abnormalities were more frequently encountered in the "IMV" group (34, 44\% of cases) than "CPAP" group (27, 24\% of cases) and "oxygen only" group $(16,25 \%$ of cases), $p$ value $=0.008$. The type of features more frequently observed were reticular in $59 \mathrm{pa}-$ tients (23\%), ground-glass opacities in $27(11 \%)$, and consolidation in 7 (3\%) of patients, Table 4.

After adjusting for demographics, comorbidities, and treatments during hospital stay (Table 5), the "IMV" group showed higher odds of DLCO impairment with respect to the "oxygen only" group although the difference was not significant (OR $=1.73,95 \%$ CI: $0.75 ; 3.99)$. No significant difference in DLCO impairment was also observed among "CPAP" and "oxygen alone" $(\mathrm{OR}=0.72$, $95 \%$ CI: $0.34 ; 1.54)$. Interestingly, in subjects treated with prophylactic heparin, the odds of DLCO alteration were 
Table 3. PFTs at 6 months from hospital discharge

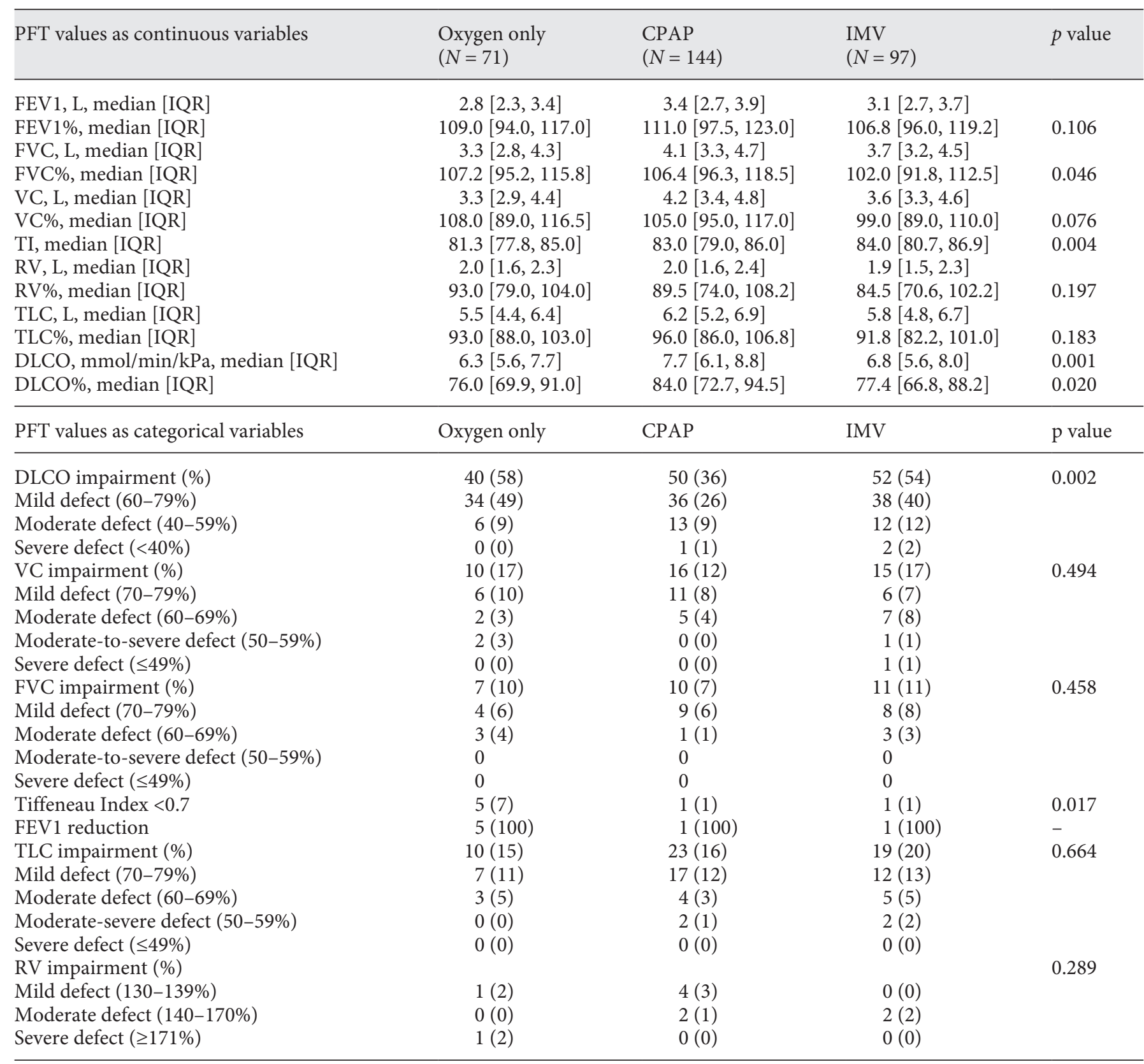

CPAP, continuous positive airway pressure; DLCO, diffusion capacity for carbon monoxide; FEV1, forced expiratory volume in the 1st second; FVC, forced vital capacity; IMV, invasive mechanical ventilation; RV, residual volume; TI, Tiffeneau Index (FEV1/FVC ratio); TLC, total lung capacity; VC, vital capacity; PFTs, pulmonary function tests; IQR, interquartile range.

halved $(\mathrm{OR}=0.45,95 \% \mathrm{CI}: 0.25 ; 0.83)$. Patients with asthma presented higher odds of altered DLCO $(\mathrm{OR}=4.86$, $95 \%$ CI: $1.09 ; 21.68)$. No difference among the 3 groups was observed in a sensitivity analysis, including smoking in the model (not included in main analysis due to the low number of active smokers ( $n=15)$ and $16 \%$ of missing).
The odds of radiological alterations on chest X-ray were higher in older patients (OR $=1.05,95 \%$ CI: $1.01 ; 1.09)$ and in patients treated with "IMV" with respect to patients on oxygen alone (OR $=3.9,95 \% \mathrm{CI}$ : $1.38 ; 11.01)$, while patients treated with CPAP did not show significantly higher odds of radiological alterations. 
Table 4. 6MWT, lung auscultation, chest X-ray, and mMRC scale results at 6 months from hospital discharge

\begin{tabular}{|c|c|c|c|c|}
\hline 6MWT & $\begin{array}{l}\text { Oxygen only } \\
(N=71)\end{array}$ & $\begin{array}{l}\text { CPAP } \\
(N=144)\end{array}$ & $\begin{array}{l}\text { IMV } \\
(N=97)\end{array}$ & $p$ value \\
\hline Metres, median [IQR] & $450[400,500]$ & $473[424.5,522.5]$ & $485[406.2,540]$ & 0.148 \\
\hline \multirow[t]{2}{*}{ Distance lower than expected (\%) } & $10(18)$ & $21(17)$ & $15(17)$ & 0.955 \\
\hline & Oxygen only & CPAP & IMV & $\mathrm{p}$ value \\
\hline \multicolumn{5}{|l|}{ Lung auscultation } \\
\hline "Velcro" crackles (\%) & $3(4)$ & $6(4)$ & $12(12)$ & 0.038 \\
\hline Wheezing (\%) & $0(0)$ & $5(3)$ & $0(0)$ & 0.070 \\
\hline \multicolumn{5}{|l|}{ Chest $X$-ray results } \\
\hline Abnormal (\%) & $16(25)$ & $27(24)$ & $34(44)$ & 0.008 \\
\hline Consolidations (\%) & $0(0)$ & $1(1)$ & $6(8)$ & 0.006 \\
\hline Ground-glass opacities (\%) & $10(16)$ & $8(7)$ & $9(12)$ & 0.186 \\
\hline Reticulations (\%) & $12(19)$ & $21(19)$ & $26(34)$ & 0.042 \\
\hline \multicolumn{5}{|l|}{$m M R C$ scale } \\
\hline \multicolumn{5}{|l|}{ Scale $(\%)$} \\
\hline 0 & $41(67)$ & $90(68)$ & $62(69)$ & \multirow{5}{*}{0.911} \\
\hline 1 & $15(25)$ & $26(20)$ & $22(24)$ & \\
\hline 2 & $3(5)$ & $11(8)$ & $5(6)$ & \\
\hline 3 & $2(3)$ & $4(3)$ & $1(1)$ & \\
\hline 4 & $0(0)$ & $1(1)$ & $0(0)$ & \\
\hline
\end{tabular}

CPAP, continuous positive airway pressure; IMV, invasive mechanical ventilation; $\mathrm{mMRC}$, Modified Medical Research Council; 6MWT, six-min walking test; IQR, interquartile range.

Fig. 2. Distribution of obstructive and restrictive ventilatory patterns according to study group. Restrictive ventilatory impairment was defined as a reduction in TLC with a normal FEV1/VC. CPAP, continuous positive airway pressure; IMV, invasive mechanical ventilation; TLC, total lung capacity; FEV1, forced expiratory volume in the 1st second; VC, vital capacity.

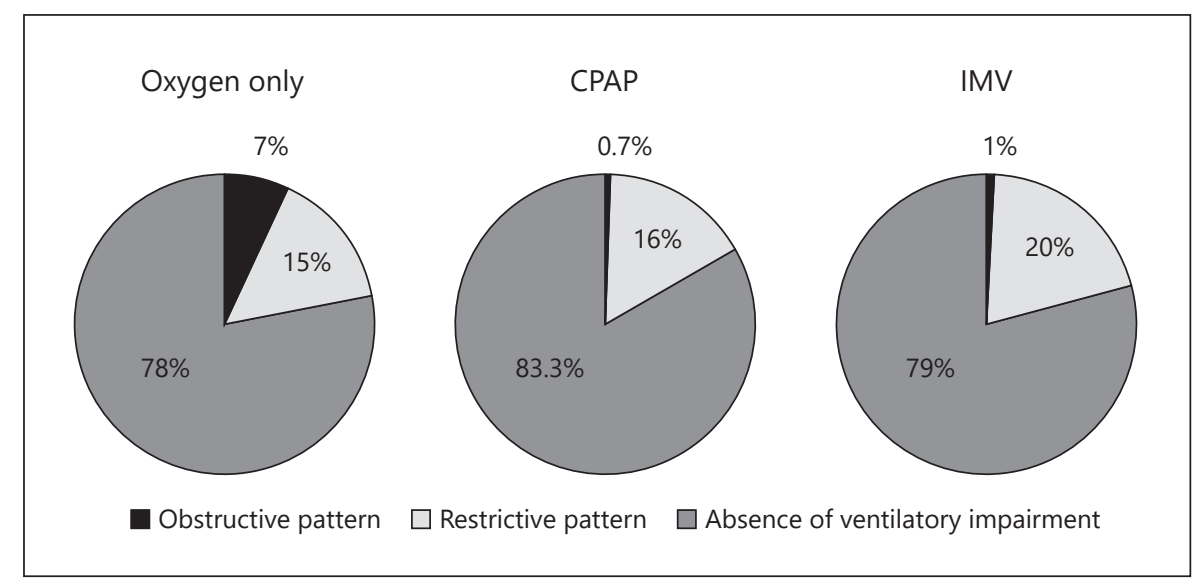

\section{Discussion}

We report the prevalence and degree of 6-month pulmonary sequelae in a cohort of 312 patients hospitalized for COVID-19 in 7 hospitals in Lombardy, the Italian region most populated and most severely affected by the pandemic so far. We observed a considerable percentage of pulmonary sequelae when considering DLCO and chest X-ray. Up to $58 \%$ and $44 \%$ of patients according to study groups showed alterations of DLCO and chest Xray, respectively. This was particularly evident in patients requiring IMV, but in the majority of cases, the degree of impairment was mild.

Patients in the "oxygen only" group showed, together with the "IMV" group, the highest degree of DLCO impairment. We speculate that this may be partially related to the 
Table 5. Multivariable logistic model results on the association between groups and DLCO or chest X-ray impairment adjusted for prespecified variables

\begin{tabular}{|c|c|c|c|c|}
\hline & OR & \multicolumn{2}{|c|}{$95 \% \mathrm{CI}$} & $p$ value \\
\hline \multicolumn{5}{|l|}{ DLCO impairment ${ }^{\wedge}$} \\
\hline CPAP versus oxygen alone & 0.72 & 0.34 & 1.54 & 0.3977 \\
\hline IMV versus oxygen alone & 1.73 & 0.75 & 3.99 & 0.1981 \\
\hline AGE, per year in males & 1.04 & 1.00 & 1.08 & $*$ \\
\hline Age, per year in females & 0.98 & 0.93 & 1.03 & $*$ \\
\hline $\mathrm{BMI}$, per $\mathrm{kg} / \mathrm{m}^{2}$ & 0.95 & 0.88 & 1.02 & 0.1283 \\
\hline Cardiovascular diseases (yes vs. no) & 0.7 & 0.34 & 1.43 & 0.3274 \\
\hline Diabetes (yes vs. no) & 2.01 & 0.83 & 4.86 & 0.1209 \\
\hline Asthma (yes vs. no) & 4.86 & 1.09 & 21.68 & 0.0381 \\
\hline Systemic steroid (yes vs. no) & 1.07 & 0.58 & 1.97 & 0.8281 \\
\hline Prophylactic heparin (yes vs. no) & 0.45 & 0.25 & 0.83 & 0.0105 \\
\hline \multicolumn{5}{|l|}{ Chest X-ray alterations ${ }^{\#}$} \\
\hline CPAP versus oxygen alone & 1.4 & 0.53 & 3.68 & 0.4997 \\
\hline IMV versus oxygen alone & 3.9 & 1.38 & 11.01 & 0.0101 \\
\hline Age, per year & 1.05 & 1.01 & 1.09 & 0.0076 \\
\hline Gender (females vs. males) & 1.54 & 0.68 & 3.49 & 0.3058 \\
\hline $\mathrm{BMI}$, per $\mathrm{kg} / \mathrm{m}^{2}$ & 0.91 & 0.84 & 1.00 & 0.0512 \\
\hline Cardiovascular diseases (yes vs. no) & 0.64 & 0.27 & 1.53 & 0.3189 \\
\hline Diabetes (yes vs. no) & 2.44 & 0.9 & 6.59 & 0.079 \\
\hline Asthma (yes vs. no) & 2.79 & 0.58 & 13.29 & 0.1983 \\
\hline Systemic steroid (yes vs. no) & 1.56 & 0.73 & 3.32 & 0.2498 \\
\hline Prophylactic heparin (yes vs. no) & 1.02 & 0.49 & 2.15 & 0.9491 \\
\hline
\end{tabular}

BMI, body mass index; CPAP, continuous positive airway pressure; DLCO, diffusion capacity for carbon monoxide; IMV, invasive mechanical ventilation; OR, odds ratio; CI, confidence interval. ^ 242 patients included with no missing on DLCO, and variables in the model, 106 with DLCO impairment. * A significant interaction between gender and age was found in this model with $p$ value $=0.0374$. For this reason, we presented the OR of age stratified by gender. ${ }^{\#} 203$ patients included with no missing on chest X-ray and variables in the model, 51 with chest $\mathrm{X}$-ray alterations. fact that the "oxygen only" group received significantly less specific treatments during hospitalization than the other groups, particularly in regards to systemic steroid $(31 \%$ in the "oxygen only" group compared to $56 \%$ and $58 \%$ in the "CPAP" and "IMV" groups, respectively) and prophylactic heparin (31\% in the "oxygen only" group compared to $47 \%$ and $59 \%$ in the "CPAP" and "IMV" groups, respectively). This could have influenced a slower recovery of normal lung anatomy and function at medium term follow-up. Thus, these preliminary observations need to be confirmed with longer follow-ups. After adjusting for potential confounders, such as treatment, DLCO impairment resulted higher for "IMV" group with respect to "oxygen only," although with not a significant difference.

DLCO can be altered both by parenchymal and pulmonary vascular diseases, and COVID-19 may have a course characterized by an overlap between interstitial pneumonia with oedema and altered pulmonary perfusion with microthrombosis and macrothrombosis [20]. Therefore, DLCO appears to be the most sensitive parameter among those available to monitor patients with COVID-19 during follow-up. Furthermore, on multivariable analysis, the use of prophylactic heparin during hospitalization appeared to act as a protective factor on the development of DLCO impairment at 6-month follow-up. The potential beneficial effect of heparin during COVID-19 acute phase has been widely discussed, although randomized clinical trials are needed [21]. The mechanisms involved include the antiviral and anti-inflammatory effect and the anticoagulant action on the hypercoagulability state associated with the disease. Our observations seem to suggest a prominent role of the vascular involvement during the acute phase of $\mathrm{CO}$ VID-19 on the onset of DLCO impairment.

In our cohort, the need for invasive ventilatory support, which may be considered a proxy of disease severity, was a risk factor for the detection of chest imaging abnormalities at 6 months. The main alterations observed were reticulations and ground-glass opacities. In particular, reticulations were significantly more frequent in patients who underwent IMV. 
Our results nicely fit with a report on 736 patients from Wuhan followed up at 6 months after acute SARS-CoV-2 infection [10]. This study showed a DLCO and radiological impairment in up to $56 \%$ and $54 \%$, respectively, of patients requiring high-flow nasal cannula, non-invasive ventilation, or IMV, and the severity of the acute disease was the major risk factor for the development of pulmonary sequelae.

Among the main strengths of our study, we acknowledge the following: (1) the multicentric design, which included both university and non-university hospitals, allowed us to increase the study cohort and to enhance the generalizability of the results; (2) the selection criteria chosen excluded patients with pre-existing structural lung diseases and those who developed bacterial and/or fungal pulmonary infections during hospitalization, which may have caused PFT and/or radiological alterations not attributable to COVID-19.

Our study also presents some limitations: (1) the study visits were conducted during the second pandemic wave and this may have contributed to the lost to follow-up of some patients that were afraid of going to the hospital for medical visits; however, the distribution of age and gender was similar among all patients recruited and patients actually visited; (2) data on the severity of radiological involvement during hospitalization, which may have had an impact on the development of pulmonary sequelae, were not collected, although the maximum ventilatory support needed by the patients gave us an hint about the severity of pneumonia.

\section{Conclusions}

Up to $58 \%$ of patients with COVID-19, according to the study group, present pulmonary sequelae, although of mild entity in the majority of cases, at 6-month follow-up. DLCO and radiological assessment appear to be the most sensitive tool to monitor patients with COVID-19 during follow-up. The need for invasive ventilatory support during hospitalization is a risk factor for detection of radiological abnormalities, but not for DLCO impairment, at follow-up. While the use of prophylactic heparin acts as a protective factor on the development of DLCO impairment.

Future studies should evaluate the pulmonary sequelae developed by patients in the second and subsequent pandemic waves to assess the impact of standard-of-care therapies, such as steroid and heparin that were not routinely used during the first wave. Furthermore, we await data on long-term sequelae with data at 1-year follow-up.

\section{Acknowledgments}

We acknowledge Davide Gaudesi, $\mathrm{PhD}$, and Silvia Mori, $\mathrm{PhD}$, from Bicocca Clinical Research Organization (BiCRO) for their support in designing eCRF and project management. We acknowledge that this research was partially supported by the Italian Ministry of University and Research (MIUR) - Department of Excellence project PREMIA (PREcision MedIcine Approach: bringing biomarker research to clinic). We acknowledge Valentina Bonfanti, Pietro Curci, Giovanni Franco, and Tommaso Passerella from University of Milano Bicocca for their support in follow-up organization and conduction. Available pre-print version of the manuscript (DOI number: 10.1101/2021.03.29.21254151).

\section{Statement of Ethics}

This study received ethics committee approval (ASST Monza, 3389 , May 21, 2020) and was conducted ethically in accordance with the World Medical Association Declaration of Helsinki. All patients provided written informed consent at the time of enrolment.

\section{Conflict of Interest Statement}

The authors have no conflicts of interest to declare.

\section{Funding Sources}

The authors have no funding to declare.

\section{Authors Contributions}

A.P. is the guarantor of this research. P.F., F.L., P.R., and A.P. were responsible for study concept and design. P.F., S.B., A.S., M.C., L.P., A.M., F.B., B.D.B., M.B., F.D.G., P.S., E.O., A.S., L.B., P.C., D.M., S.H., and A.C. contributed to patient recruitment and follow-up. All authors contributed to data acquisition. P.F., S.B., P.R., S.G., M.G.V., and L.Z. performed data analysis. P.F., F.L., P.R., and S.B. contributed to the drafting of this manuscript. All authors read and approved the final manuscript.

\section{Data Availability Statement}

Individual participant data referring to this article (i.e., text, tables and figures) will be made available upon reasonable request. The study protocol will be made available for researchers who provide a methodologically sound proposal. Proposals should be directed to paola.faverio@unimib.it.
Faverio et al. 


\section{References}

1 Guan WJ, Ni ZY, Hu Y, Liang WH, Ou CQ, $\mathrm{He}$ JX, et al. Clinical Characteristics of coronavirus disease 2019 in China. N Engl J Med. 2020;382:1708-20.

2 Tian S, Hu W, Niu L, Liu H, Xu H, Xiao SY. Pulmonary pathology of early-phase 2019 novel coronavirus (COVID-19) pneumonia in two patients with lung cancer. J Thorac Oncol. 2020;15(5):700-4.

3 Mineo G, Ciccarese F, Modolon C, Landini MP, Valentino M, Zompatori M. Post-ARDS pulmonary fibrosis in patients with $\mathrm{H} 1 \mathrm{~N} 1$ pneumonia: role of followup CT. Radiol Med. 2012;117(2):185-200.

4 Zhang P, Li J, Liu H, Han N, Ju J, Kou Y, et al. Long-term bone and lung consequences associated with hospital-acquired severe acute respiratory syndrome: a 15-year follow-up from a prospective cohort study. Bone Res. 2020;8:8.

5 Zhang R, Pan Y, Fanelli V, Wu S, Luo AA, Islam D, et al. Mechanical stress and the induction of lung fibrosis via the midkine signaling pathway. Am J Respir Crit Care Med. 2015; 192(3):315-23.

6 Cabrera-Benitez NE, Laffey JG, Parotto M, Spieth PM, Villar J, Zhang H, et al. Mechanical ventilation-associated lung fibrosis in acute respiratory distress syndrome: a significant contributor to poor outcome. Anesthesiology. 2014;121(1):189-98.

7 Zhao YM, Shang YM, Song WB, Li QQ, Xie $\mathrm{H}, \mathrm{Xu} \mathrm{QF}$, et al. Follow-up study of the pulmonary function and related physiological characteristics of COVID-19 survivors three months after recovery. EClinicalMedicine. 2020;25:100463.
8 van Gassel RJJ, Bels JLM, Raafs A, van Bussel BCT, van de Poll MCG, Simons SO, et al. High prevalence of pulmonary sequelae at 3 months after hospital discharge in mechanically ventilated survivors of COVID-19. Am J Respir Crit Care Med. 2021;203(3):371-4.

9 van den Borst B, Peters JB, Brink M, Schoon Y, Bleeker-Rovers CP, Schers H, et al. Comprehensive health assessment three months after recovery from acute COVID-19. Clin Infect Dis. 2020:21.

10 Huang C, Huang L, Wang Y, Li X, Ren L, Gu $\mathrm{X}$, et al. 6-month consequences of COVID-19 in patients discharged from hospital: a cohort study. Lancet. 2021;397(10270):220-32.

11 Pfeifer M, Ewig S, Voshaar T, Randerath WJ, Bauer T, Geiseler J, et al. Position paper for the state-of-the-art application of respiratory support in patients with COVID-19. Respiration. 2020;99(6):521-42.

12 von Elm E, Altman DG, Egger M, Pocock SJ, Gøtzsche PC, Vandenbroucke JP, et al. The strengthening the reporting of observational studies in epidemiology (STROBE) statement: guidelines for reporting observational studies. Int J Surg. 2007;12(12):1495-9.

13 Graham BL, Steenbruggen I, Miller MR, Barjaktarevic IZ, Cooper BG, Hall GL, et al. Standardization of spirometry 2019 update. An official American thoracic society and european respiratory society technical statement. Am J Respir Crit Care Med. 2019;200(8):e7088.

14 Graham BL, Brusasco V, Burgos F, Cooper BG, Jensen R, Kendrick A, et al. Executive summary: 2017 ERS/ATS standards for single-breath carbon monoxide uptake in the lung. Eur Respir J. 2017 Jan;49(1):1600016.
15 Quanjer PH, Stanojevic S, Cole TJ, Baur X, Hall GL, Culver BH, et al. Multi-ethnic reference values for spirometry for the 3-95-yr age range: the global lung function 2012 equations. Eur Respir J. 2012;40:1324-43.

16 Holland AE, Spruit MA, Troosters T, Puhan MA, Pepin V, Saey D, et al. Official European respiratory society/American thoracic society technical standard: field walking tests in chronic respiratory disease. Eur Respir J. 2014;44(6):1428-46.

17 Enright PL, Sherrill DL. Reference equations for the six-minute walk in healthy adults. Am J Respir Crit Care Med. 1998;158(5 Pt 1): 1384-7.

18 Carsin AE, Fuertes E, Schaffner E, Jarvis D, Antó JM, Heinrich J, et al. Restrictive spirometry pattern is associated with low physical activity levels. A population based international study. Respir Med. 2019 Jan;146:116-23.

19 Pellegrino R, Viegi G, Brusasco V, Crapo RO, Burgos F, Casaburi R, et al. Interpretative strategies for lung function tests. Eur Respir J. 2005;26:948-68.

20 Camporota L, Vasques F, Sanderson B, Barrett NA, Gattinoni L. Identification of pathophysiological patterns for triage and respiratory support in COVID-19. Lancet Respir Med. 2020;8(8):752-4.

21 Hippensteel JA, LaRiviere WB, Colbert JF, Langouët-Astrié CJ, Schmidt EP. Heparin as a therapy for COVID-19: current evidence and future possibilities. Am J Physiol Lung Cell Mol Physiol. 2020;319(2):L211-7. 